\section{Über die Fixirung des Chroms durch die Wollfaser.}

Von

\section{Dr. Edmund Knecht.}

Seit der Einführung des Kaliumbichromats als Wollbeize um das Jahr 1840 ist verhältnissmässig wenig dazu beigetragen worden, um die genaue Menge des auf der Faser fixirten Chroms, sowie die Natur der chemischen Veränderungen, die während des gewöhnlichen Beizprocesses ror sich gehen, festzustellen.

In den Lehrbüchern (vgl, besonders Hummel: The dying of textile fabries S. 208 und 326 ), sowie in technischen Blättern $^{1}$ ) ist dieser Gegenstand allerdings von Zeit zu Zeit berührt worden: da sich aber die Schlussfolgerungen überall auf allgemeine Beobachtungen und nicht auf die Ergebnisse streng quantitativer Versuche stützen, so können die daraus abgeleiteten Theorien keinen hohen wissenschaftlichen Werth besitzen.

Das bei diesen Versuchen angewandte Material bestand in einer mittleren Sorte gewaschenen und gewalkten Flanells, der Bequemlichkeit wegen in Stücke zerschnitten, deren jedes $10 \mathrm{~g}$ wog. Die Wolle enthielt 0,60 Proc. Asche.

a) Menge des fixirten Chroms. Beim ersten Versuche wurden 6 Stücke 1 Stunde lang bei $100^{\circ}$ in Lösungen von doppelt chromsauren Kali gebeizt, die je 100 cc betrugen. Die Beize wurde in folgenden Verhältnissen angewendet:

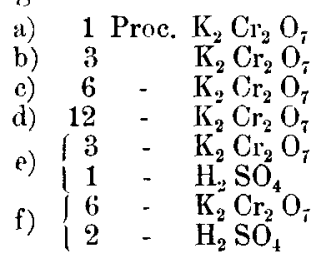

Nach dem Beizen wurden die Stücke herausgenommell, der Überschuss an absorbirter Flüssigkeit möglichst gut ausgequetscht und wieder in die Beizelösung gebracht. Die Stücke wurden dann in kaltem Wasser gut ausgewaschen und getrocknet.

Bei jedem einzelnen Versuche wurde sodann die Wolle in einer Platinschale eingeäschert, mit Ätzkali und chlorsaurem Kali (um alles $\mathrm{Cr}_{2} \mathrm{O}_{3}$ in $\mathrm{K}_{2} \mathrm{Cr}_{4}$ überzuführen) geschmolzen, dann in Wasser aufgelöst und Zehntelnormal Ferrosulfat-Lösung im Über-

1) Vgl. J. B. Wilkins on: Journ. Soc. Dyers \& Colourists Bd. 2 S. 36 und R. Lloyd Whiteley: J. Ch. Ind. 1887, 131. schuss zugesetzt; der Überschuss wurde mit Zehntelnormal Kaliumbichromat zurücktitrirt und auf diese Weise die Menge des auf der Faser fixirten Chroms (als $\mathrm{K}_{2} \mathrm{Cr}_{2} \mathrm{O}_{7}$ berechnet) bestimmt.

Um die Menge des in der Lösung zurückbleibenden Chroms festzusetzen, liess man die alten Beizelösungen sich abkühlen, verdünnte sie je auf 1000 cc und bestimmte die Menge des Chroms (als $\mathrm{K}_{2} \mathrm{Cr}_{2} \mathrm{O}_{7}$ ) in einem abgemessenen Theile mit Hülfe von Zehntelnormal Ferrosulfatlösung und Kaliumbichromat.

Folgende Zahlen stellen die absoluten Mengen von Chrom, als $\mathrm{K}_{2} \mathrm{Cr}_{2} \mathrm{O}_{7}$ berechnet, dar, welche zum Theil auf der Faser fixirt, zum Theil in der Lösung geblieben waren.

\begin{tabular}{c|c|c|c}
\hline & In Lösung & Auf der Faser & Verlust \\
\hline & $g$ & $g$ & $\mathrm{~g}$ \\
$\mathrm{a}$ & $\mathbf{0 , 0 3 5 4}$ & 0,0565 & 0,0081 \\
$\mathrm{~b}$ & $\mathbf{0 , 1 7 7 0}$ & 0,0987 & 0,0245 \\
$\mathrm{c}$ & 0,4320 & $\mathbf{0 , 1 6 2 6}$ & 0,0054 \\
$\mathrm{~d}$ & 0,9826 & $\mathbf{0 , 1 8 5 8}$ & 0,0316 \\
$\mathrm{e}$ & $\mathbf{0 , 1 6 4 6}$ & $\mathbf{0 , 1 2 8 7}$ & 0,0069 \\
$\mathrm{f}$ & $\mathbf{0 . 3 1 9 4}$ & $\mathbf{0 , 1 7 8 2}$ & $0, \mathbf{1 0 2 4}$
\end{tabular}

Da die Menge des angewandten Materials je $10 \mathrm{~g}$ betrug, so erhält man den Procentsatz von fixirtem Kaliumbichromat einfach durch Multiplication obiger Zahlen mit 10. Der in der Tabelle angedeutete Verlust rührt höchstwahrscheinlich davon her, dass das gelöste Kaliumbichromat durch die Wolle theilweise zu Chromoxydsalz reducirt wurde.

In der zweiten Versuchsreihe wurde einerseits die Menge von Chrom (als $\mathrm{K}_{2} \mathrm{Cr}_{2} \mathrm{O}_{7}$ berechnet) bestimmt, die aus Natriumbichromat fixirt wird, und zwar aus Mengenverhältnissen des letzteren, die beziehungsweise 3 und 6 Proc. Kaliumbichromat äquivalent sind; andererseits auch der Einfluss von Weinstein bei der Anwendung dieser Beizen untersucht.

$\mathrm{Zu}$ diesen Zwecke wurden 4 Stücke genau wie oben in folgenden Procentsätzen gebeizt

\begin{tabular}{|c|c|c|}
\hline & Proc. & \\
\hline & 2,67 & $\mathrm{Na}_{2} \mathrm{Cr}_{2} \mathrm{O}_{7}$ \\
\hline & $\{2,67$ & $\mathrm{Na}_{2} \mathrm{Cr}_{2} \mathrm{C}$ \\
\hline & & einstei \\
\hline & 18 & Weinstei \\
\hline
\end{tabular}

Beim Ausfübren dieser Bestimmungen wurde das nämliche Verfahren und dieselbe Vorsicht wie bei der ersten Versuchsreihe beobachtet. Wo Weinstein in Anwendung kam, wurde die Bestimmung des in Lösung bleibenden Bichromates wegen der stark reducirenden Wirkung des ersteren weggelassen. Es wurden folgende Zablen erhalten: 


\begin{tabular}{|c|c|}
\hline In Lösung & Anf der Faser \\
\hline & $\stackrel{g}{g}$ \\
\hline a) 0,1965 & 0,0826 \\
\hline b) 0,4323 & 0,0983 \\
\hline c) - & 0,0855 \\
\hline d) & 0,0934 \\
\hline
\end{tabular}

Da Andere bei mehreren früheren Untersuchungen auf die grossen Resultatsunterschiede aufmerksam gemacht haben, die man beim Beizen mit äquivalenten Mengen von Bichromat und Chromoxydsetzen erhält, wurde in einer dritten Versuchsreihe die Menge von Chrom bestimmt, die sich aus Chromalaun fixirt, wenn dieser im Äquivalent von beziehungsweise 3 und 6 Proc. Kaliumbichromat angewendet wird:

\begin{tabular}{|c|c|}
\hline \multirow[b]{2}{*}{ Proc. Chromalaum } & $\begin{array}{l}\text { Anf der Faser } \\
\text { fixirte Menge } \\
\left.\text { (als } \mathrm{K}_{2} \mathrm{Cr}_{2} \mathrm{O}_{i}\right)\end{array}$ \\
\hline & \\
\hline a) 10,30 & 0,1916 \\
\hline b) 20,61 & 0,3184 \\
\hline c) $\left\{\begin{array}{c}10,30 \\
3 \text { Proc. }\end{array}\right.$ & ein 0,0920 \\
\hline
\end{tabular}

Einfluss der Zeit. Um den Einfluss der Zeit auf die Menge des fixirten Chroms zu untersuchen, wurden 6 Stücke Flanell mit Abänderung der Zeitdauer in siedende Lösungen von 3 Proc. Kaliumbichromat eingetaucht, mit folgenden Resultaten:

\begin{tabular}{c|c|c|c}
\hline & $\begin{array}{c}\text { Dauer der } \\
\text { Eintauchung }\end{array}$ & $\begin{array}{c}\text { In Iësung } \\
\text { bleibende } \\
\text { Mengen }\end{array}$ & $\begin{array}{c}\text { Anf der Faser } \\
\text { fixirte Mengen } \\
\text { (nach den Diffe- } \\
\text { renzen) }\end{array}$ \\
\hline & Ninuten & $\mathrm{g}$ & $\mathrm{g}$ \\
$\mathrm{a}$ & $\mathbf{1 0}$ & 0,2506 & 0,0494 \\
$\mathrm{~b}$ & 20 & 0,2456 & 0,0541 \\
$\mathrm{e}$ & 30 & 0,2113 & 0,0887 \\
$\mathrm{~d}$ & 40 & 0,1818 & 0,1182 \\
$\mathrm{e}$ & 50 & 0,1818 & 0,1182 \\
$\mathrm{f}$ & 60 & 0,1780 & 0,1220
\end{tabular}

Einfluss der Wärme. Beim Beizen mit Kaliumbichromat ist bekanntlich der Einfluss der Wärme von grosser Bedeutung, und es werden die besten Resultate bei Anwendung einer bohen Temperatur erzielt. Dies bestätigt sich durch folgende zwei bezw. bei 80 und $100^{\circ}$ ausgeführten Versuche

$\begin{array}{ccc}\text { Temperatur } & \begin{array}{c}\text { In Lösung bleibende } \\ \text { Menge } g\end{array} & \begin{array}{c}\text { Auf der Faser } \\ \text { ixirte Menge (durch } \\ \text { Differenzen) }\end{array} \\ 80^{\circ} & 0,2310 & 0,0690 \\ 100^{\circ} & 0,1768 & 0,1232\end{array}$

Menge von Chrom, die bei wiederholter Auffrischung desselben Färbebades fixirt wird. Um das Verfahren des "Chromens", wie dasselbe praktisch in den Färbereien ausgeführt wird, so genau wie möglich nachzuahmen, wurde folgende Reihe von Versuchen gemacht:

Stücke wollenen Materials wurde in gewöhnlicher Weise mit 3 Proc. $\mathrm{K}_{2} \mathrm{Cr}_{2} \mathrm{O}_{7}$, sowie mit 3 Proc. $\mathrm{K}_{2} \mathrm{Cr}_{2} \mathrm{O}_{7}$ nebst 1 Proc. $\mathrm{H}_{2} \mathrm{~S} \mathrm{O}_{*}$ gebeizt, und die Mengen des in der
Lösung bleibenden und des auf der Faser fixirten Chroms bestimmt.

Dieselbe Behandlung wurde nun zum zweiten und zum dritten Male unter jedesmaligem Zusatz der gleichen Procentsätze Beize wiederholt (indem aber zugleich dafür Sorge getragen wurde die zur Bestimmung herausgenommene Menge $\mathrm{K}_{2} \mathrm{Cr}_{2} \mathrm{O}_{7}$ durch einen äquivalenten Zusatz zu ersetzen).

Diese Versuche lieferten folgende interessante Resultate:

\begin{tabular}{ccc}
\multicolumn{3}{c}{ I. Beize: $3 \% \mathrm{~K}_{2} \mathrm{Cr}_{2} \mathrm{O}_{7}$} \\
In Lösung $\mathrm{g}$ & Auf der Faser $\mathrm{g}$ \\
$1 \mathrm{mal}$ & 0,1770 & 0,0987 \\
$2-$ & 0,3243 & 0,1523 \\
$3-$ & 0,4275 & 0,1893 \\
II. Beize: & $3 \% \mathrm{~K}_{2} \mathrm{Cr}_{2} \mathrm{O}_{7}, \mathbf{1 \%} \mathrm{H}_{2} \mathrm{SO}_{4}$ \\
\multicolumn{4}{c}{ In Lösung g } & Auf der Faser g \\
$1 \mathrm{mal}$ & 0,1646 & 0,1287 \\
$2-$ & 0,2653 & 0,1920 \\
$3-$ & 0,3214 & 0,2240
\end{tabular}

b) Bestimmung des Zustandes in welchem das Chrom fixirt wird.

Es ist schon mehrmals versucht worden, die Fixirung des Chroms aus Kaliumbichromat auf der Wollfaser zu erklären; die aufgestellten Theorien stützen sich aber leider meistens auf allgemeine Beobachtungen, und beruhen nicht auf streng wissenschaftlichen Gründen. Nach einer vorherrschenden 'Meinung soll das Bichromat durch die reducirende Wirkung der Wolle zersetzt und in Chromoxydhydrat verwandelt werden, das sich auf der Faser festsetzt. Obschon diese Ansicht in gewissen Fällen mit der Erfahrung übereinstimmt, wie $z$. B. wenn Weinstein in Gemeinschaft mit dem Bichromat angewendet wird, so gilt dieselbe nicht für Anwendung von Bichromat für sich allein, wenn nicht das Kochen sehr lange fortgesetzt wird. Nach einer anderen von $R$. Lloyd Whiteley (D. Ch. Ind. 1887, 131) ausgesprochenen Ansicht ist das Fixiren des Bichromats eine einfache mechanische Absorption des Salzes durch die Wollfaser. Dass Chromsäure in einer oder der andern Form absorbirt wird, scheint unzweifelhaft, aber folgende Versuche beweisen, dass die Absorption auch von einer chemischen Veränderung in der Beizelösung begleitet wird.

$\mathrm{Z}$ wei Stücke Flanell wurden in reinem destillirtem Wasser gewaschen, um alle $\mathrm{Un}^{-}$ reinigkeiten, die etwa aus den Operationen des Waschens und Walkens hätten herrühren können, zu entfernen. Eines dieser Stücke wurde sodann mit 10 Proc. $\mathrm{K}_{2} \mathrm{Cr}_{2} \mathrm{O}_{3}$ gebeizt, das andere aber während der gleichen Zeit in destillirtem Wasser gekocht. Die Lösungen wurden dann mittels Lackmoid geprüft. Während in der Abknchung der Wolle in 
reinem Wasser keine bemerkbare Veränderung eintrat, färbte sich dagegen die das Bichromat entbaltende Lösung entschieden blau. Da das doppelt chromsaure Kali sich zu Lackmoid neutral verhält, das einfachchromsaure Kali aber alkalisch, so würden die Resultate dieses Versuches darauf hindeuten, dass während des Beizevorganges eine Zersetzung des doppeltchromsauren Kalis in chromsaures Kali und Chromsäure stattgefunden hat; ersteres bleibt in der Lösung während die Chromsäure auf der Wollfaser fixirt wird. Die von der Wolle fixirte Menge und die relativen Mengen von Chromat und Bichromat, die in Lösung bleiben, wurden bestimmt und sind durch folgende Zahlen ausgedrückt:

$$
\text { In Lösting : }
$$

Als $\mathrm{K}_{2} \mathrm{Cr} \mathrm{O} \ldots 0,2916 \mathrm{~g}$ Als $\mathrm{K}_{2} \mathrm{Cr}_{2} \mathrm{O}_{7} \ldots 0,4749$ -

Auf der Faser: $0,2970 \mathrm{~g}$

(berechnet aus $\mathrm{K}_{2} \mathrm{Cr} \mathrm{O}_{7}$ )

Dass diese Veränderungen auch im Grossen stattfinden, erhellt aus folgender Analyse einer angeblich 15 aufeinanderfolgende Male in der Praxis gebrauchten Beizelösung.

$$
\text { im } \ell
$$

$$
\begin{array}{ll}
\mathrm{K}_{2} \mathrm{Cr}_{2} \mathrm{O}_{7} & 1,177 \mathrm{~g} \\
\mathrm{~K}_{2} \mathrm{CrO}_{4} & 1,672-
\end{array}
$$

Relative Werthe vou chromsaurem Kali, doppeltchromsaurem Kali und Chromsäure als Wollbeizen. Drei Stücke Flanell wurden mit Kaliumchromat, Kaliumbichromat und Chromsäure gebeizt, wobei in jedem Fall Mengen angewendet wurden, die 3 Proc. Bichromat äquivalent waren. Das auf der Faser fixirte Chrom wurde bestimmt, und es ergaben sich folgende als Chromsäure ausgedrückte Mengen:

$\begin{array}{ll}\text { Chromat } & 0,0605 \mathrm{~g} \\ \text { Bichromat } & 0,0805 \text { - } \\ \text { Chromsäure } & 0,1658 \text { - }\end{array}$

Schlussfolgerungen. 1) Die Menge vom Chrom, die beim Beizen mit Kaliumbichromat auf der Wollfaser fixirt wird, verändert sich mit der Concentration der Lösung. Unter den in der ersten Versuchsreihe beobachteten Bedingungen ist das Verhältniss des fixirten Chroms (als Bichromat berechnet) zu der angewandten Menge annähernd

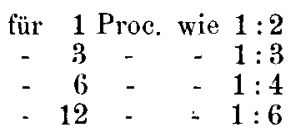

Wird neben dem Bichromat zugleich Schwefelsäure angewendet, so ist die fixirte Menge beträchtlich grösser und hängt ebenfalls ron der Concentration der Lösung ab, wie aus folgenden Zahlen hervorgeht:

$$
\begin{gathered}
\text { für } 3 \text { Proc. wie } 2: 5 \\
-6-\quad-1: 3
\end{gathered}
$$

Es ist bemerkeuswerth, dass beim Beizen mit 3 Proc. Bichromat, wie es gewöhnlich im grossen Maassstabe geschieht, nur etwa 1 Proc. auf der Faser fixirt wird.

2) Bei Anwendung von reinem Natriumbichromat, äquivalent 3 Proc. Kaliumbichromat, wird eine verhältnissmässig grössere Menge Chrom fixirt, als bei Anwendung des Kalisalzes. Dieser Umstand erklärt vielleicht zum Theil die verschiedenen Resultate, die man in der Praxis mit diesen zwei Beizen erhält.

3) Ein Zusatz von Weinstein zu der Beize hat keinen wesentlichen Einfluss auf die Menge des fixirten Chroms.

4) Die Menge ron Chrom aus Chromalaun fixirt, ist ungefähr zweimal so gross, wie die aus äquivalenten Procenten Bichromat fixirte Menge. In diesem Falle ist das Chrom wahrscheinlich nicht so innig mit der Faser verbunden, wie wenn Bichromat als Beize benutzt wird, was ans den schlechten Färberesultaten, die man mit Chromalaun gebeizter Wolle erhält, hervorgeht.

5) Beim Beizen mit Kaliumbichromat ist die Menge des aufgenommenen Chroms in directem Verhältniss mit der Zeitdaner des Eintauchens und mit der Temperatur des Bades.

6) Bei der praktischen Benutzung eines Bichromatbades muss die Menge des in demselben enthaltenen Chroms nothwendig mit der Anzahl der in demselben Bade vorgenommenen Operationen zunehmen. Werden z. B. für das erste Bad 3 Proc. Bichromat angewendet, so absorbirt die Wolle 1 Proc., während 2 Proc. in der Lösung bleiben. Nach Zusatz von 3 Proc. für das zweite Bad, hat man im Ganzen 5 Proc. Bichromat in der Lösung, woron 1/3 wieder von der Wolle absorbirt wird, so dass noch $5-5 / 3=10 / 3$ Proc. Bichromat in Lösung bleiben. In dieser Weise uruss das Bad sich nothwendig so sehr concentriren, dass es endlich entleert werden muss, wobei täglich grosse Mengen Bichromat unausweichlich verloren gehen. Theoretisch wäre der rationellste Weg des Beizens mit Bichromat der, dass man 3 oder 4 Proc. des Salzes zum ersten Bade benutzt, und hierauf jedem nachfolgenden Bade gerade soviel neues Bichromat zusetzt als ron der Wolle aufgenommen wurde (d. h. ungefähr 1 Proc.) zugleich mit einer genügenden Menge Schwefelsäure, um das sich bildende chromsaure Kali zu neutralisiren, bez. wieder in Bichromat zu verwandeln.

7) Das Fixiren von Chrom auf Wolle beim Beizen mit Kaliumbichromat ist immer von einer theilweisen Zersetzung des Bades in chromsaures Salz und Chromsäure beglei- 
tet. Obschon die Reaction nicht quantitative, und von einem gew issen Maass mechanischer Absorption begleitet ist, so ist es doch nicht unwahrscheinlich, dass das Keratin oder die Substanz der Wolle dabei die Rolle einer Base übernimmt, die mit Chromsäure eine unlösliche oder wenig lösliche Verbindung eingeht.

8) Betrachtet man die Menge des auf der Faser fixirten Chroms als das Maass der Leistungsfähigkeit der Beize, so wäre die Chromsäure weitaus die beste Chrombeize für Wolle. Ihr zunächst im Werth steht Kaliumbichromat in Gemeinschaft mit Schwefelsäure, dann Kaliumbichromat allein und zuletzt das chromsaure Kali.

An dieser Stelle möchte ich meinen beiden Schùlern Herren $P$. Kay und E. Bastow für die Ausführung obiger Versuche meinen wärmsten Dank aussprechen.

Bradford Technical College.

August 1887 .

\section{Brennstoffe und Beleuchtung.}

Zur Destillation von Kohle, Schiefer u. dgl. will O. Rose in London ( ${ }^{*}$ D.R.P. No. 40212) liegende Retorten verwenden, durch welche die betr. Stoffe mittels Schnecke ununterbrochen hindurchgeschoben werden.

O R uppert in Hamm $\left({ }^{*}\right.$ D.R.P. No. 40030$)$ beschreibt Gasvorlagen und Gasreiniger (*D.R P. No. 40262 u. 40263 ).

Zur Verwendung des Steinkohlentheeres zur Herstellung von Leuchtgas destillirt W. Burns (Iron. 29, 383) den Theer unter Einblasen von Wasserdampf und fuhrt das Dampfgemisch durch Retorten, welche mit glühenden Holzkohlen gefüllt siud. $1 l$ Theer und $2 \mathrm{k}$ Wasserdampf sollen 4 cbm Leuchtgas von 16 Kerzen geben.

Burns behauptet ferner, $1 \mathrm{t}$ gewöhnliche Steinkohle, bei niederer Temperatur unter Druck in Theer (und Ammoniak) übergeführt und dann in obiger Weise zu Leuchtgas verarbeitet gebe $600 \mathrm{cbm}$ Leuchtgas (Angaben deren Richtigkeit doch wohl zu bezweifeln ist).

Der Gasretorten ofen mit Wärmespeicher von Fr. Siemens (Engl. P. 1886 No. 815i) Leichnet sich durch das Vorhandensein einer wagrechten Verbrennungskammer aus. Fig. 93 zeigt denselben im Schnitt halb nach $A-B$, halb nach $C-F$, Fig. 94 halb nach $G-K L-N O$, halb nach $G-K P O$, Fig. 95 nach Q-R. Die bei $g$ eintretende Luft steigt in den von aussen erhitzten Kanälen $h$ auf und trifft bei $k$ mit dem in den Ge-
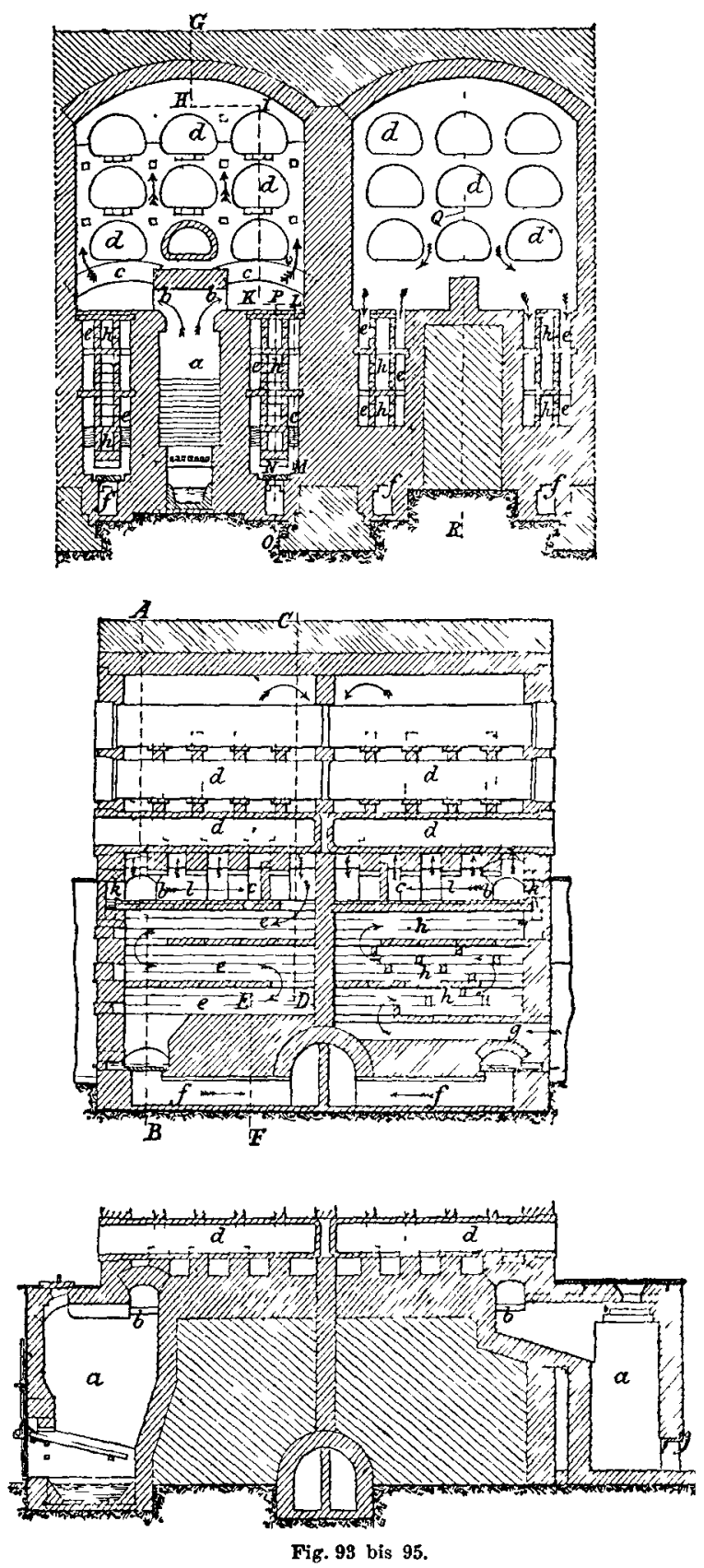

nerator $a$ erzeugten und durch Kanal b $<u^{-}$ geführten Heizgase zusammen. Die Flamme geht von dem Heizkanale $l$ aus durch das durchbrochene Gewölbe $c$, um die Retorten $d$ herum, dann wieder nach unten, um durch die Kanäle $e$ und $f$ Lu entweichen.

Zur Auffindung undichter Stellen in Gasleitungen bringt E. Rattier (J. éclaiı. 1887. $\left.{ }^{*} 167\right)$ durch eine Bohrung 